\title{
A distinct bone phenotype in ADPKD patients with end-stage renal disease
}

\begin{abstract}
Pieter Evenepoel ${ }^{1}$, Kathleen Claes ${ }^{1}$, Etienne Cavalier ${ }^{2}$, Bjorn Meijers ${ }^{1}$, Peter Stenvinkel ${ }^{3}$, Geert Behets ${ }^{4}$, Magdalena Jankowska ${ }^{5}$, Patrick D'Haese ${ }^{4}$ and Bert Bammens ${ }^{1}$

${ }^{1} \mathrm{KU}$ Leuven Department of Microbiology and Immunology, Laboratory of Nephrology; University Hospitals Leuven, Department of Nephrology, Leuven, Belgium; ${ }^{2}$ Clinical Chemistry, Université de Liège, Belgium; ${ }^{3}$ Department of Renal Medicine, Karolinska University Hospital, Stockholm, Sweden; ${ }^{4}$ Department of Biomedical Sciences, Laboratory of Pathophysiology, Antwerp University, Belgium; and ${ }^{5}$ Department of Nephrology, Transplantology and Internal Medicine, Medical University of Gdańsk, Poland
\end{abstract}

Autosomal dominant polycystic kidney disease (ADPKD) is among the most common hereditary nephropathies. Low bone turnover osteopenia has been reported in mice with conditional deletion of the PKD1 and PKD2 genes in osteoblasts, and preliminary clinical data also suggest suppressed bone turnover in patients with ADPKD. The present study compared the bone phenotype between patients with end stage renal disease (ESRD) due to ADPKD and controls with ESRD due to other causes. Laboratory parameters of bone mineral metabolism (fibroblast growth factor 23 and sclerostin), bone turnover markers (bone alkaline phosphatase, tartrate-resistant acid phosphatase $5 b)$ and bone mineral density (BMD, by dual energy $x$-ray absorptiometry, DXA) were assessed in 518 patients with ESRD, including 99 with ADPKD. Bone histomorphometry data were available in 71 patients, including 10 with ADPKD. Circulating levels of bone alkaline phosphatase were significantly lower in patients with ADPKD (17.4 vs $22.6 \mathrm{ng} / \mathrm{mL}$ ), as were histomorphometric parameters of bone formation. Associations between ADPKD and parameters of bone formation persisted after adjustment for classical determinants including parathyroid hormone, age, and sex. BMD was higher in skeletal sites rich in cortical bone in patients with ADPKD compared to nonADPKD patients (Z-score midshaft radius -0.04 vs -0.14 ; femoral neck -0.72 vs -1.02 ). Circulating sclerostin levels were significantly higher in ADPKD patients ( $2.20 \mathrm{vs}$ $1.84 \mathrm{ng} / \mathrm{L}$ ). In conclusion, patients with ESRD due to ADPKD present a distinct bone and mineral phenotype, characterized by suppressed bone turnover, better preserved cortical BMD, and high sclerostin levels.

Kidney International (2019) 95, 412-419; https://doi.org/10.1016/ j.kint.2018.09.018

KEYWORDS: ADPKD; bone; mineral metabolism

Copyright (C) 2019, International Society of Nephrology. Published by Elsevier Inc. All rights reserved.

Correspondence: Pieter Evenepoel, Division of Nephrology, University Hospital Leuven, Herestraat 49, B-3000 Leuven, Belgium. E-mail: Pieter.Evenepoel@uz.kuleuven.ac.be

Received 9 July 2018; revised 6 September 2018; accepted 13 September 2018

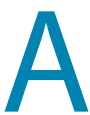

utosomal-dominant polycystic kidney disease (ADPKD) is an inherited disorder that commonly results in renal failure in humans; ADPKD accounts for $7 \%$ to $10 \%$ of patients with end-stage renal disease (ESRD) ${ }^{1,2}$ More than $85 \%$ of patients with ADPKD have mutations in PKD1, PKD2, or both. ${ }^{1,3}$ PKD1 encodes polycystin (PC)1, which functions as a $\mathrm{G}$ protein-coupled receptor. ${ }^{4}$ $P K D 2$ encodes PC2, which is a receptor-activated calcium channel. ${ }^{1,5}$ PC1 interacts with PC2 to form heterodimers to colocalize in the primary cilia through interactions between the C-terminus of $\mathrm{PC} 2$ and kinesin family member $3 \mathrm{~A}$ (KIF3A). The primary cilium is a solitary, immotile microtubule-based extension present on nearly every mammalian cell. This organelle has established mechanosensory roles in several contexts including kidney, liver, and the embryonic node. ${ }^{6,7}$ It is postulated that the primary cilium plays a key role in normal physiologic functions of renal epithelia and that defects in ciliary function may contribute to the pathogenesis of ADPKD. ${ }^{8}$ Recent research has implicated the primary cilium as a mechanosensor in bone as well. ${ }^{9-11}$ Primary cilia not only play a role in embryonal skeletogenesis but also in postnatal/adult bone homeostasis. Osteocytes, that is, the most numerous bone cells, express the PC1/PC2 complex and exhibit a dendritic structure with extensive connectivity throughout the mineralized matrix of bone. The precise molecular mechanisms whereby osteocytes respond to and convert mechanical stimuli to biochemical signals remain elusive.

Because mechanical loading is the primary functional determinant of bone mass and architecture and a dysfunctional ciliary PC1/PC2 complex may disturb mechanosensation and transduction, it may be hypothesized that ADPKD may associate with a specific bone phenotype. Several lines of experimental and clinical evidence support this hypothesis. Heterozygous PKD1 mutant mice have a decreased bone mineral density, trabecular bone volume, and cortical thickness. These mice also have downregulated gene expression of the osteoblastic markers Runx2, osterix, and osteocalcin, as well as an increase of the osteoprotegerin to receptor activator of nuclear factor- $\mathrm{KB}$ ligand ratio. ${ }^{12}$ Along with this finding, Gitomer et al. ${ }^{13}$ observed dramatically decreased bone formation in a small bone biopsy study including 5 patients with ADPKD who had preserved renal function. Gitomer et al. ${ }^{14}$ 
Table 1 | Demographics and parameters of mineral metabolism in ESRD patients with and without ADPKD

\begin{tabular}{|c|c|c|c|}
\hline Demographics and laboratory parameters & ADPKD $(n=99)$ & Non-ADPKD $(n=419)$ & $P$ value \\
\hline Age, yr & $56.9 \pm 8.8$ & $54.2 \pm 13.5$ & 0.5 \\
\hline Male sex, $\%$ & 49.5 & 63.3 & 0.02 \\
\hline $\mathrm{BMI}, \mathrm{kg} / \mathrm{m}^{2}$ & $24.7 \pm 4.0$ & $24.9 \pm 4.3$ & 0.8 \\
\hline Renal diagnosis, $\%$ & & & $<0.0001$ \\
\hline Diabetic nephropathy & 0 & 10.7 & \\
\hline Glomerulonephritis/vasculitis & 0 & 31.5 & \\
\hline Cystic/hereditary/congenital diseases & 100 & 5.5 & \\
\hline Miscellaneous & 0 & 8.4 & \\
\hline Etiology unknown or missing & 0 & 25.6 & \\
\hline Diabetes mellitus, $\%$ & 5.1 & 21.2 & 0.0002 \\
\hline CVD, \% & 27.3 & 42.3 & 0.005 \\
\hline PTX, \% & 7.1 & 14.6 & 0.05 \\
\hline $25(\mathrm{OH}) \mathrm{D}_{3}, \mu \mathrm{g} / \mathrm{l}$ & $37.7(25.1-49.2)$ & $35.5(23.6-48.5)$ & 0.3 \\
\hline $1.25(\mathrm{OH})_{2} \mathrm{D}_{3}, \mathrm{ng} / \mathrm{l}$ & $27.3(20.2-34.1)$ & $26.7(17.9-37.5)$ & 1 \\
\hline FGF23, ng/l & 3323 (1083-9548) & $2040(606-7573)$ & 0.04 \\
\hline Sclerostin, ng/l & $2.20(1.68-3.16)$ & $1.84(1.28-2.57)$ & 0.001 \\
\hline OPG, pmol/l & $9.97(8.0-12.3)$ & $10.2(7.3-14.0)$ & 0.7 \\
\hline sRANKL, pmol/l & $0.075(0.063-0.14)$ & $0.097(0.063-0.17)$ & 0.01 \\
\hline sRANKL/OPG & $0.009(0.006-0.016)$ & $0.010(0.005-0.021)$ & 0.2 \\
\hline C-reactive protein, mg/l & $3.60(1.50-8.30)$ & $3.30(1.30-7.50)$ & 0.6 \\
\hline $\mathrm{IL}-6, \mathrm{pg} / \mathrm{ml}$ & $1.71(0.87-2.77)$ & $1.35(0.63-2.37)$ & $<0.05$ \\
\hline $\mathrm{tAP}, \times \mathrm{UNL}$ & $0.72(0.53-0.95)$ & $0.80(0.62-1.09)$ & 0.03 \\
\hline BsAP, ng/ml & $17.4(13.2-27.0)$ & $22.6(16.1-35.5)$ & $<0.0001$ \\
\hline PINP, $\mu \mathrm{g} / \mathrm{l}$ & $77.9(49.8-111.1)$ & $83.6(53.7-143.1)$ & 0.1 \\
\hline TRAP5b, U/I & $4.65(3.13-6.57)$ & $5.46(3.84-7.59)$ & 0.006 \\
\hline
\end{tabular}

ADPKD, autosomal-dominant polycystic kidney disease; BMI, body mass index; biPTH, biointact parathyroid hormone; BsAP, bone-specific alkaline phosphatase; ESRD, endstage renal disease; FGF23, fibroblast growth factor 23; IL-6, interleukin-6; OPG, osteoprotegerin; P1NP, procollagen type I N propeptide; PTX, parathyroidectomy; sRANKL, soluble receptor activator of nuclear factor-KB ligand; TRAP5b, tartrate-resistant acid phosphatase $5 \mathrm{~b}$.

Data are presented as mean $\pm \mathrm{SD}$ or median (interquartile range).

also reported a lower areal bone mineral density (aBMD) in patients with early stage ADPKD compared with healthy control subjects.

The present observational study aimed to confirm and extend these findings. Laboratory parameters of bone metabolism and turnover, bone mineral density (BMD), and bone histomorphometry were investigated in a large cohort of patients with ESRD who were being referred for kidney transplantation.

\section{RESULTS}

\section{Demographics}

Five hundred eighteen patients with ESRD, all kidney transplant candidates, were enrolled in the present study. ADPKD was the primary renal disease in 99 patients, corresponding to a prevalence of $19 \%$. Table 1 compares demographics between patients with and without ADPKD. Females were more prevalent among patients with ADPKD. Furthermore, patients with ADPKD had fewer diagnoses of diabetes and cardiovascular morbidity and a borderline significant lower history of parathyroidectomy. Fractures were equally prevalent in patients with and without ADPKD.
Bone turnover markers and laboratory parameters of mineral metabolism

Bone-specific alkaline phosphatase (BsAP) and tartrateresistant acid phosphatase 5b (TRAP5b) levels were significantly lower in patients with ADPKD than in patients without ADPKD (Table 1; Figure 1). Bone turnover markers strongly correlated with each other $(\rho \geq 0.5$, all $P<0.0001$, Supplementary Table S1). Importantly, in multivariable regression analyses, ADPKD was identified as determinant of circulating BsAP and TRAP5b levels, independent of age, sex, diabetes, parathyroid hormone (PTH), fibroblast growth factor 23 (FGF23), and sclerostin. Serum phosphate, sclerostin, and FGF23 levels were significantly higher in patients with ADPKD compared with patients without ADPKD (Table 1). In multivariable regression analysis, age, sex, dialysis vintage, $\mathrm{PTH}$, FGF23, and calcitriol, as well as diagnosis of ADPKD, were identified as independent determinants of circulating sclerostin levels, explaining $24 \%$ of its variability. Determinants of FGF23 were quite different. Only calcium, phosphate, and calcitriol were retained in the final model, altogether explaining $44 \%$ of the variability of FGF23 (Table 2). 

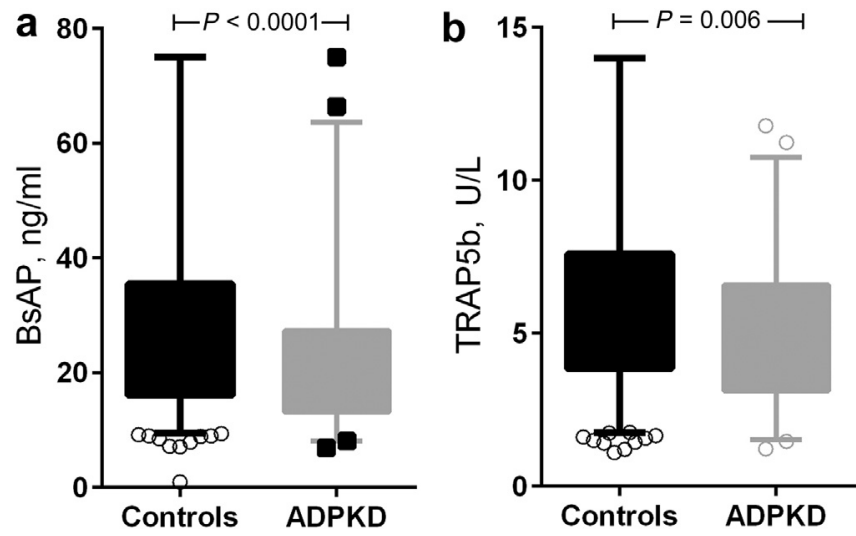

Figure 1 | Levels of bone-specific alkaline phosphatase (BsAP) (a) and tartrate-resistant acid phosphatase 5b (TRAP5b) (b) in patients with end-stage renal disease due to autosomaldominant polycystic kidney disease (ADPKD) versus control subjects without ADPKD.

\section{ADPKD and bone histomorphometry}

Bone biopsies were performed in 90 patients at the time of transplantation and yielded bone specimens of sufficient quality to perform quantitative bone histomorphometry in 71 patients (ADPKD, $n=10$; non-ADPKD, $n=61$; Table 3 ). Inadequate samples were equally distributed between the 2 groups. Bone volume did not differ between patients with and without ADPKD. Mineralization tended to be higher in patients with ADPKD. Static parameters of bone turnover were lower in patients with ADPKD. However, statistically significant differences were reached for bone formation (osteoblast perimeter/tissue perimeter) only.

\section{ADPKD and aBMD}

Table 4 presents aBMD in patients with and without ADPKD. Median $Z$ scores, expressing the SD relative to age- and sexmatched control subjects, were below zero across all skeletal sites examined in both groups, confirming that ESRD is a state of low bone mineral density. $Z$ scores were higher in patients with ADPKD, with significance reached both at the mid-shaft radius and femoral neck. Of note, results were not meaningfully affected by the exclusion of patients who had undergone parathyroidectomy.

\section{DISCUSSION}

The main finding of the present cross-sectional observational study is that patients with ADPKD who have ESRD show a distinct bone phenotype characterized by suppressed bone turnover and preserved aBMD.

The gold standard for quantifying bone turnover is bone histomorphometry. Bone histomorphometry provides information not only on bone turnover but also on bone volume and mineralization. In the present study, static bone histomorphometric data were available in 71 patients and showed a trend of decreased bone turnover and increased mineralization in patients with ADPKD. Probably because of limited power, significance was reached for osteoblast perimeter/tissue perimeter $(P=0.04)$, that is, a marker of bone formation, only. These data confirm data from a pilot bone biopsy study in patients with early stage ADPKD. ${ }^{13}$ They also align with radiologic data from more than 4 decades ago showing suppressed bone erosion in patients with ADPKD who were treated with hemodialysis compared with patients without ADPKD who were treated with hemodialysis. ${ }^{15}$ Obtaining a

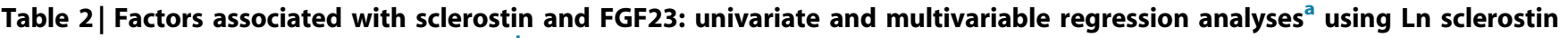
and Ln FGF23 as the dependent variable

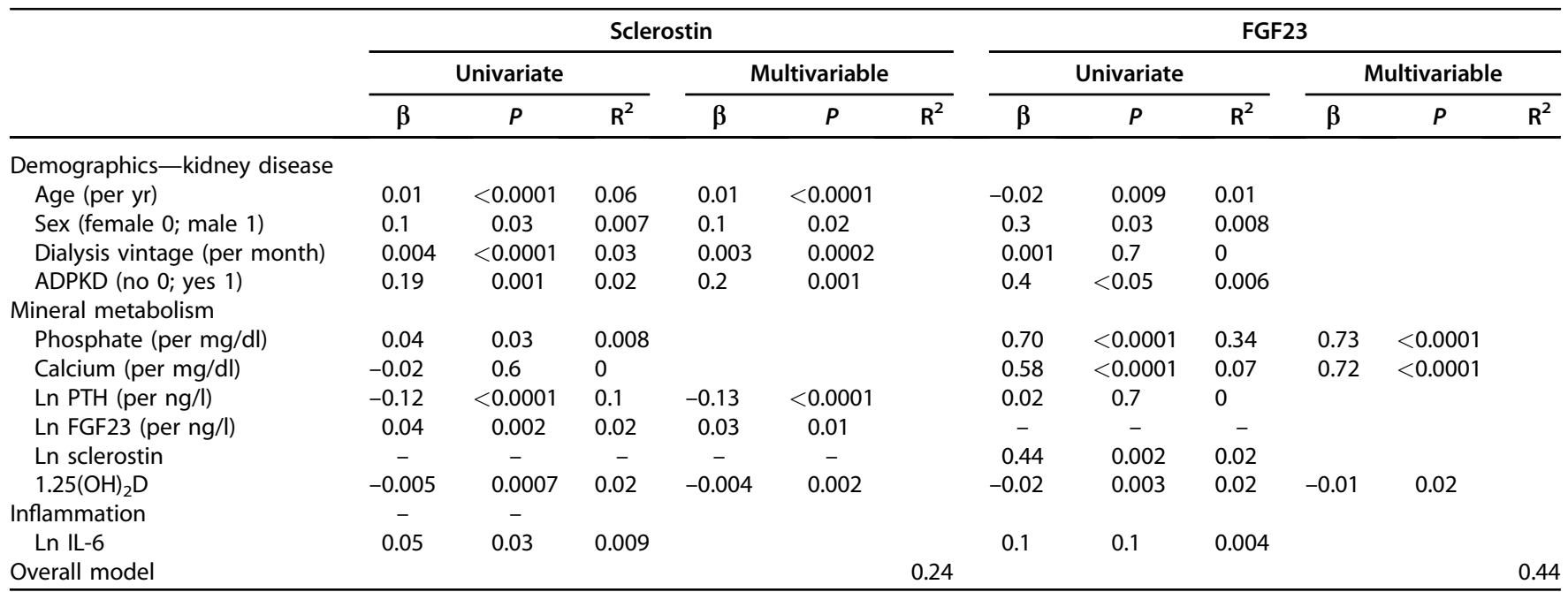

ADPKD, autosomal-dominant polycystic kidney disease; FGF23, fibroblast growth factor 23; IL-6, interleukin-6; Ln, natural logarithmic; PTH, parathyroid hormone. Parameters studied were age, sex, diabetes, ADPKD, dialysis vintage, Ln PTH, Ln FGF23, and Ln sclerostin. Only parameters univariately associated at $P \leq 0.2$ are mentioned in the table.

${ }^{\mathrm{a}}$ Generalized linear model.

${ }^{b}$ Because collinearity, only bone alkaline phosphatase was included in the multivariable model. Findings were similar for procollagen type I $\mathrm{N}$ propeptide and tartrate-resistant acid phosphatase $5 \mathrm{~b}$ (data not shown). 
Table 3 | Key demographics, laboratory parameters, and bone histomorphometry data in ESRD patients with and without ADPKD

\begin{tabular}{|c|c|c|c|}
\hline & $\operatorname{ADPKD}(n=10)$ & $\begin{array}{l}\text { Non-ADPKD } \\
(n=61)\end{array}$ & $P$ value \\
\hline \multicolumn{4}{|l|}{ Demographics } \\
\hline Age, yr & $59.2 \pm 10.7$ & $54.4 \pm 13.1$ & 0.4 \\
\hline BMI, $\mathrm{kg} / \mathrm{m}^{2}$ & $28.2 \pm 7.6$ & $25.5 \pm 4.2$ & 0.4 \\
\hline \multicolumn{4}{|l|}{$\begin{array}{l}\text { Laboratory } \\
\text { parameters }\end{array}$} \\
\hline Calcium, mg/dl & $9.5 \pm 0.5$ & $9.3 \pm 0.7$ & 0.7 \\
\hline Phosphate, mg/dl & $5.3 \pm 1.1$ & $4.4 \pm 1.4$ & $<0.05$ \\
\hline biPTH, ng/l & $197.3(112.0-210.8)$ & $204.4(99.0-315.9)$ & 0.5 \\
\hline FGF23, ng/l & $5231(1544-15913)$ & $1159(427-5245)$ & 0.02 \\
\hline Sclerostin, ng/l & $1.90(1.68-2.97)$ & $1.58(1.07-2.28)$ & $<0.05$ \\
\hline $\mathrm{BsAP}, \mathrm{ng} / \mathrm{ml}$ & $17.4(14.2-22.1)$ & $20.4(15.3-35.5)$ & 0.2 \\
\hline PINP, $\mu \mathrm{g} / \mathrm{l}$ & $83.0(63.7-89.1)$ & $80.0(53.0-131.2)$ & 0.6 \\
\hline TRAP $5 \mathrm{~b}, \mathrm{U} / \mathrm{l}$ & $4.34(3.26-6.43)$ & $5.80(4.34-7.86)$ & 0.2 \\
\hline \multicolumn{4}{|l|}{$\begin{array}{l}\text { Bone } \\
\text { histomorphometry }\end{array}$} \\
\hline B.Ar/T.Ar, \% & $19.1(14.4-23.1)$ & $21.8(17.5-26.5)$ & 0.2 \\
\hline O.Ar/B.Ar, \% & $1.13(0.85-1.60)$ & $2.05(1.11-3.14)$ & 0.08 \\
\hline O.Pm/B.Pm, \% & $11.2(8.40-19.3)$ & $20.1(11.5-25.4)$ & 0.06 \\
\hline $\mathrm{O} . \mathrm{Wi}, \mu \mathrm{m}$ & $6.72(6.14-8.37)$ & $7.41(6.41-9.41)$ & 0.3 \\
\hline Ob.Pm/O.Pm, \% & $0.00(0.00-6.86)$ & $9.56(0.00-19.9)$ & 0.08 \\
\hline Ob.Pm/T.Pm, \% & $0.00(0.00-1.27)$ & $1.61(0.00-4.04)$ & 0.04 \\
\hline E.Pm/B.Pm, \% & $4.10(2.00-5.32)$ & $4.23(2.69-7.45)$ & 0.3 \\
\hline Oc.Pm/E.Pm, \% & $10.8(0.00-21.4)$ & $16.6(0.00-22.6)$ & 0.6 \\
\hline Oc.Pm/T.Pm, \% & $0.31(0.00-1.09)$ & $1.61(0.00-4.04)$ & 0.4 \\
\hline Tb.th, $\mu \mathrm{m}$ & 135.5 (109.2-165.4) & $145.4(126.4-168.0)$ & 0.3 \\
\hline Tb.N, $\mathrm{mm}^{-1}$ & $1.97(1.65-2.27)$ & $1.70(1.47-2.00)$ & 0.3 \\
\hline Tb.Sp, $\mu \mathrm{m}$ & $481.1(365.4-515.1)$ & $372.9(292.7-456.2)$ & 0.2 \\
\hline
\end{tabular}

ADPKD, autosomal-dominant polycystic kidney disease; B.Ar, bone area; biPTH, biointact parathyroid hormone; $\mathrm{BMI}$, body mass index; $\mathrm{B} . \mathrm{Pm}$, bone perimeter; $\mathrm{BsAP}$, bone-specific alkaline phosphatase; E.Pm, eroded perimeter; ESRD, end-stage renal disease; FGF23, fibroblast growth factor 23; M mineralization; O.Ar, osteoid area; Ob.Pm, osteoblast perimeter; Oc.PM, osteoclast perimeter; O.Pm, osteoid perimeter; O.Wi: osteoid width; PINP, procollagen type I N propeptide; T, turnover; T.Ar, tissue area; Tb.N, trabecular number; Tb.Sp, trabecular spacing; Tb.th, trabecular thickness; Tb.Wi, trabecular width; TRAP5b, tartrate-resistant acid phosphatase $5 \mathrm{~b}$; Tt.Pm, total perimeter; $\mathrm{V}$, volume.

Data are presented as mean \pm SD or median (interquartile range).

bone biopsy specimen is invasive and requires the necessary skills; furthermore, analyzing bone biopsy specimens is expensive and necessitates specific histopathologic expertise that is not widely available. Therefore, a bone biopsy is not feasible in all patients all of the time. ${ }^{16}$ Noninvasive imaging techniques (including isotope techniques) ${ }^{17}$ and bone turnover markers have been suggested as a surrogate of or adjuvant to bone biopsy to assess bone turnover. In the present study, we assessed bone turnover by measuring circulating levels of BsAP, trimeric N-terminal propeptide of type I collagen (PINP), and TRAP5b, because these analytes are stable and undergo little degradation, are not cleared by the kidneys, exert little circadian rhythm, and are not affected by food intake. BsAP (-30\%) and TRAP5b $(-17 \%)$ were significantly lower in patients with ADPKD, whereas PINP (-7\%) was only nominally lower in patients with ADPKD. The apparent discrepancy between BsAP and PINP remains to be explained but could be related to limitations inherent to the biomarker. Thus far, BsAP, PINP, and TRAP5b are not used routinely in clinical practice. In the absence of frank liver dysfunction, the total alkaline phosphatase level may be a valid alternative. In agreement with previous cohort studies in patients with early ${ }^{18}$ and advanced ${ }^{19}$ stage renal disease, we observed suppressed total alkaline phosphatase levels in patients with ADPKD. Of interest, a low total alkaline phosphatase level recently has been shown to be independently associated with a higher height-corrected total kidney volume in patients with early $\mathrm{ADPKD}^{20}$ and thus might qualify as a biomarker of disease severity.

Of interest, these clinical observations perfectly align with data from recent in vitro and animal studies using advanced genetic approaches. Xiao and Quarles ${ }^{10}$ demonstrated decreased osteoblast-mediated bone formation along with decreased expression of osteoblast-related genes including Runx2, Osteocalcin, Osteopontin, SOST, and FGF23 in mice with conditionally and selectively deleted PKD1 and PKD2 in osteoblasts or osteocytes. Moreover, these mice showed significant reductions in both serum concentrations and bone mRNA expression of receptor activator of nuclear factor $-\kappa B$ ligand and TRAP, whereas serum PTH and osteoprotegerin did not differ from the wild-type mice. Altogether, these experimental data support the concept that primary cilium/ polycystin complex plays an important role in bone mechanosensing. The precise mechanisms involved in translating mechanical signals into (re)modeling response remain unclear. Mounting evidence points to Wnt signaling pathway components, and the antiosteogenic canonical Wnt inhibitor Sost/sclerostin in particular, as important players in regulating the bone's adaptive response to loading. Wnt- $\beta$-catenin signaling directly affects both the osteoblast and the osteoclast bone cell lineages and also indirectly affects these cells through cross talk in the bone environment, inducing an overall increase in osteoblastogenesis together with a decrease in osteoclastogenesis. ${ }^{21}$ Experimental and clinical evidence demonstrated that bone sclerostin expression and circulating sclerostin levels increased during skeletal mechanical unloading. $^{21,22}$ Starting from the premise that a disrupted mechanosensation mimics in some way the condition of unloading, increased bone sclerostin expression and higher circulating sclerostin levels would be anticipated in persons with ADPKD. This was actually observed in the present study and in a previous similar but smaller cohort study. ${ }^{19}$ Importantly, $\mathrm{ADPKD}$ is associated with higher circulating sclerostin levels, independent of classic determinants, including PTH, age, sex, and inflammation. Remarkably, in aforementioned mice models of conditional deleted polycystins, bone sclerostin mRNA was not increased but suppressed. Residual confounding, assay related limitations, and altered translation all may be hypothesized to contribute to the discrepancy. If increased protein expression is confirmed in persons with $\mathrm{ADPKD}$, additional studies will be required to decipher the molecular pathways involved. Besides being the consequence of impaired mechanosensation, increased sclerostin levels in persons with ADPKD also could be an adaptive counterregulatory response to enhanced canonical Wnt signaling as observed in polycystic kidneys. ${ }^{23}$ Recent evidence points to high levels of hypoxia-inducible factor $1-\alpha$ as the 
Table 4 | aBMD in ESRD patients with and without ADPKD

\begin{tabular}{|c|c|c|c|}
\hline & ADPKD & Non-ADPKD & $P$ value \\
\hline \multicolumn{4}{|l|}{$\mathrm{R} 1 / 3(n=342)$} \\
\hline BMD & $0.708(0.647-0.767)$ & $0.683(0.607-0.754)$ & 0.07 \\
\hline T score & $-0.172(-0.595$ to -0.017$)$ & $-0.251(-1.070$ to -0.101$)$ & 0.03 \\
\hline $\mathrm{Nl} /$ osteopenia/osteoporosis, \% & $79.2 / 12.5 / 8.3$ & $73.7 / 15.6 / 10.7$ & 0.6 \\
\hline \multicolumn{4}{|l|}{ UDR $(n=342)$} \\
\hline $\mathrm{BMD}$ & $0.390(0.357-0.439)$ & $0.391(0.328-0.448)$ & 1.0 \\
\hline $\mathrm{Nl} /$ osteopenia/osteoporosis, \% & $32.9 / 31.4 / 35.7$ & $24.1 / 41.7 / 34.2$ & 0.2 \\
\hline \multicolumn{4}{|l|}{$\mathrm{LS}(n=518)$} \\
\hline BMD & $0.902(0.789-1.037)$ & $0.942(0.839-1.058)$ & 0.06 \\
\hline T score & $-1.880(-2.87$ to -0.450$)$ & $-1.467(-2.407$ to -0.433$)$ & 0.09 \\
\hline Z score & $-0.84(-2.02$ to 0.51$)$ & $-0.77(-1.66$ to 0.34$)$ & 0.5 \\
\hline $\mathrm{Nl} /$ osteopenia/osteoporosis, \% & $34.4 / 36.5 / 29.2$ & $39.0 / 38.5 / 22.5$ & 0.4 \\
\hline \multicolumn{4}{|l|}{$\mathrm{TH}(n=502)$} \\
\hline BMD & $0.849(0.748-0.947)$ & $0.855(0.720-0.917)$ & 0.1 \\
\hline T score & $-1.048(-1.837$ to -0.379$)$ & $-1.286(-2.025$ to -0.645$)$ & $<0.05$ \\
\hline Z score & $-1.37(-2.08$ to -0.37$)$ & $-1.25(-2.18$ to -0.55$)$ & 1.0 \\
\hline $\mathrm{Nl} /$ osteopenia/osteoporosis, \% & $47.9 / 43.8 / 8.3$ & $37.2 / 51.7 / 11.1$ & 0.1 \\
\hline
\end{tabular}

$\mathrm{aBMD}$, areal bone mineral density; ADPKD, autosomal-dominant polycystic kidney disease; BMD, bone mineral density; ESRD, end-stage renal disease; FN, femoral neck; LS, lumbar spine; $R$, radius; $R 1 / 3$. mid-shaft radius; $T H$, total hip; UDR, ultradistal radius.

Data are presented as median (interquartile range).

culprit of increased osteocytic sclerostin expression and secretion in persons with ADPKD. ${ }^{24,25}$

A body of experimental and clinical evidence indicates that sclerostin not only suppresses bone formation ${ }^{26-28}$ but also influences serum concentrations of hormones that regulate mineral accretion, including calcitriol and FGF23. ${ }^{29}$ In this regard, the observation of an independent negative association between sclerostin and calcitriol and positive association between sclerostin and FGF23 aligns with findings in SOST knockout mice. ${ }^{29}$

Mice with conditionally and selectively deleted PKD1 and PKD2 in osteoblasts or osteocytes showed a reduced BMD, trabecular bone volume, and cortical thickness. ${ }^{12}$ Also, in patients with early stage ADPKD, a lower aBMD compared with healthy control subjects has been reported. ${ }^{14}$ To the contrary, in the present study we observed a better preserved aBMD in patients with ADPKD compared with patients without ADPKD. The different stage of kidney disease probably explains this controversy (Figure 2). In the setting of advanced chronic kidney disease, ADPKD-related suppression of bone remodeling may limit hyperparathyroidism-mediated bone loss. Bone remodeling activity affects bone volume and degree of mineralization, which are both important determinants of aBMD. As a consequence of an imbalance between resorption and formation at the individual bone remodeling units, high bone turnover causes accelerated bone (volume) loss. Moreover, when bone turnover is high or increased, the probability increases that a cortical or trabecular bone structural unit will be resorbed before completion of its secondary mineralization, which leads at the tissue level to a greater proportion of younger and submaximally mineralized bone. ${ }^{30}$ In early stage chronic kidney disease, conversely, a low bone volume resulting from an imbalance between bone resorption and bone formation may be speculated to negate the impact of any pivotal benefit related to the suppression of bone turnover.

A key question is whether aforementioned alterations affect bone strength and fracture risk in patients. The present cohort study was not powered to answer this question. Clinical fractures were as prevalent in patients with ADPKD as in those without ADPKD. Notably, in a recent large population study in kidney transplant recipients, ADPKD was observed to confer an increased fracture risk, similar to diabetic nephropathy. ${ }^{31}$ In patients undergoing dialysis, on the other hand, the incident fracture rate was shown to vary according to the cause of kidney disease: patients with ADPKD had the lowest rate and patients with diabetes had the highest rate. ${ }^{32}$ Future epidemiologic studies should account for $\mathrm{ADPKD}$ as a potential confounder.

Besides increased circulating sclerostin levels, we also observed increased FGF23 levels in patients with ADPKD compared with patients without APKD. It remains to be defined whether these increased FGF23 levels result from increased skeletal or extraskeletal production. In regression analysis, the association between ADPKD and FGF23 disappeared after adjustment for serum phosphate. Serum phosphate levels were significantly higher in patients with ADPKD, even after adjustment for age, sex, and residual renal function. Previous observations in patients with early stage ADPKD support the hypothesis that the higher serum phosphate levels in persons with ADPKD might be a 

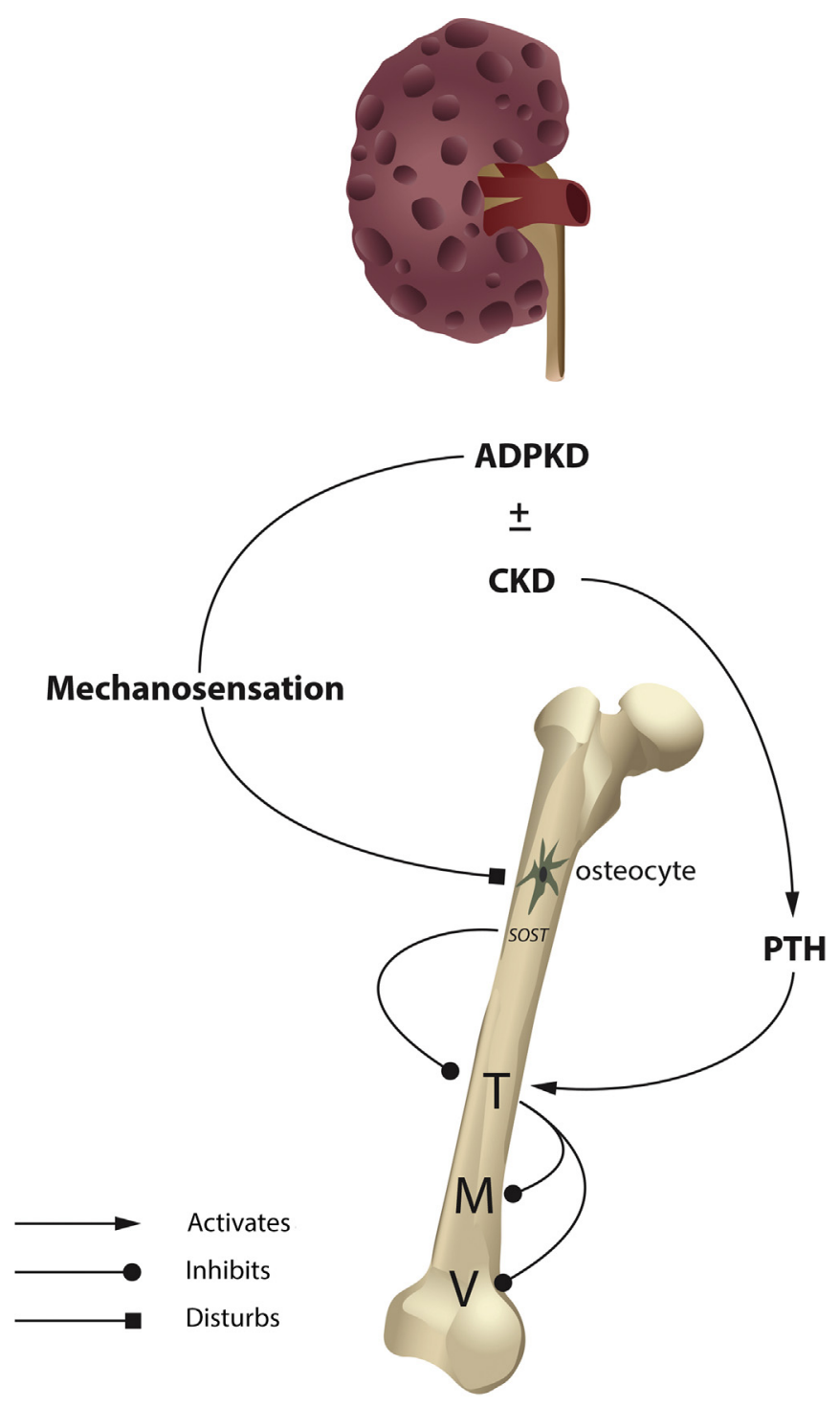

Figure 2 | Working model linking autosomal-dominant polycystic kidney disease (ADPKD) to bone phenotype according to stage of disease. In early stage disease, ADPKD associates with low bone turnover, osteopenia, probably as a consequence of disrupted mechanosensation and increased sclerostin expression. In a person with advanced stage disease, ADPKD mitigates hyperparathyroidismrelated bone mineral density loss by suppressing bone turnover. CKD, chronic kidney disease; $\mathrm{M}$, mineralization; $\mathrm{PTH}$, parathyroid hormone; $T$, turnover; $\mathrm{V}$, volume.

reflection of Klotho deficiency, thus implying FGF23 resistance. ${ }^{33}$ Additional research is needed to clarify this issue.

In conclusion, patients with ADPKD and ESRD present a specific bone phenotype characterized by suppressed bone turnover, preserved aBMD, and high sclerostin levels. Clinical implications and therapeutic consequences remain to be defined.

\section{MATERIALS AND METHODS \\ Design and study population}

This study is an ancillary analysis of data collected in the frame of other studies exploring various aspects of bone health in renal transplant candidates before and after engraftment (NCT00547040, NCT01886950).

Adult patients ( $>18$ years) with ESRD who were referred for single kidney transplantation at the University Hospital Leuven, Belgium, between 23 April 2006 and 21 December 2013 were eligible for inclusion in this cross-sectional observational study $(n=950$; Supplementary Figure S1). Only patients with an available DXA scan within 2 weeks after transplantation were included in the present analysis $(n=518)$. Baseline demographics, laboratory parameters of mineral metabolism, and aBMD data in the overall cohort have been discussed previously (other data [P. Evenepoel et al., unpublished data, 2018]). The present study focuses on differences between patients with ADPKD $(n=99)$ and without ADPKD $(n=419)$ and includes data on bone histomorphometry obtained in a subset of patients (ADPKD, $n=10$ vs. non-ADPKD, $n=61$ ). The study adhered to the principles of the Declaration of Helsinki and was approved by the Ethical Committee of KU Leuven. All patients provided written informed consent.

\section{Clinical data}

Relevant demographics, therapy (including details on mineral metabolism therapy), routine biochemistry, comorbidities, and fracture history were extracted from electronic files. Skull and digit fractures were excluded, as well as fractures associated with major trauma (e.g., motor vehicle accidents).

\section{Biochemistry}

Blood samples were collected at the time of admission for the renal transplant procedure (random, nonfasted). Samples were stored for $<2$ hours at $5{ }^{\circ} \mathrm{C}$ until centrifugation. Upon arrival at the laboratory, the blood samples were centrifuged at $3000 \mathrm{rpm}$ for 10 minutes, aliquoted, and either processed immediately or stored at $-80^{\circ} \mathrm{C}$ until analysis. Levels of creatinine, hemoglobin, total calcium, phosphate, magnesium, total alkaline phosphatase, and albumin were measured using standard laboratory techniques. $1,25(\mathrm{OH})_{2} \mathrm{VitD}$ (calcitriol), 25(OH)VitD (calcidiol), and full-length (biointact) PTH were determined by immunoradiometric assays, as described elsewhere. ${ }^{34-36}$ Total alkaline phosphatase levels were expressed as times upper normal limit to harmonize for the various assays being used for the duration of the study.

Serum sclerostin (TECOmedical, Sissach, Switzerland; reference range $[R R] 450 \pm 150,510 \pm 140$, and $590 \pm 130 \mathrm{pg} / \mathrm{ml}$ in men, premenopausal women, and postmenopausal women, respectively), biointact FGF23 (Kainos Laboratories, Inc., Tokyo, Japan; RR 8-78 pg/ml), osteoprotegerin (Biomedica, Vienna, Austria; p50 of a healthy population: $2.7 \mathrm{pmol} / \mathrm{L}$ ), and soluble receptor activator of nuclear factor- $\mathrm{KB}$ ligand (Biomedica; $\mathrm{p} 50$ of a healthy population: $0.14 \mathrm{pmol} / \mathrm{l}$ ) were measured according to the manufacturers' instructions. Interleukin-6 was measured on a MESO QuickPlex SQ120 multiplex imager (Meso Scale Discovery, Rockville, MD) using an electrochemiluminescence multiplex immunoassay (Human Proinflammatory Panel I [4-Plex], Meso Scale Discovery) according to the manufacturer's instructions. Bone-specific alkaline phosphatase (BsAP; RR 7.9-25.5 $\mu \mathrm{g} / \mathrm{l}$ in men and 6.1-22.2 and 7.1-23.9 $\mu \mathrm{g} / \mathrm{l}$ in pre- and postmenopausal women, respectively), trimeric ("intact") PINP (RR 12.8-71.9 $\mu \mathrm{g} / \mathrm{l}$ in men and 13.7-71.1 and $<82.6 \mu \mathrm{g} / \mathrm{l}$ in premenopausal and postmenopausal women, respectively) and TRAP5b (RR 1.4-6.1 U/l in men and 1.2-4.8 and 1.1-6.9 U/l in pre- and postmenopausal women, respectively) were measured with the IDS iSYS instrument (IDS, Boldon, UK). These cut-offs are obviously method dependent because large intermethod 
variation has been observed in patients with chronic kidney disease. ${ }^{37}$ All the coefficients of variation of the assays used in this study were $<10 \%$.

\section{Bone densitometry}

Measurements of aBMD were performed within 2 weeks after transplantation by DXA using a Hologic Discovery densitometer (Hologic QDR-4500A, Hologic, Marlborough, MA) at the lumbar spine (L1 through L4, $n=518)$, total hip $(n=502)$, and femoral neck $(n=502)$. In a subset of patients, aBMD $(n=342)$ also was assessed at the radius of the nondominant arm, both mid-shaft (R1/3) and ultradistal. All DXA scans were analyzed by a single certified and highly experienced operator. Results were expressed as absolute BMD $\left(\mathrm{g} / \mathrm{cm}^{2}\right)$, as $\mathrm{T}$ score (SD relative to 20- to 30-year-old white U.S. women according to the National Health and Nutrition Examination Survey reference), and as Z score (SD relative to age- and sex-matched control subjects). Osteopenia was defined as a T score between -1 and -2.4 and osteoporosis was defined as a $\mathrm{T}$ score of -2.5 and below.

\section{Bone histomorphometry}

In a subset of 90 patients, a bone biopsy was performed at the end of the kidney transplant procedure using a needle with an internal diameter of $4.5 \mathrm{~mm}$ (Osteobell, Biopsybell, Mirandola, Italy) at a site $2 \mathrm{~cm}$ posterior and $2 \mathrm{~cm}$ inferior to the anterior iliac spine. Because the timing of deceased donor kidney transplantation is unpredictable, bone biopsies at the time of transplantation were performed without prior double tetracycline labeling. The method for quantitative histomorphometry of bone has been described elsewhere. ${ }^{38}$ Briefly, biopsy specimens were fixed in ethanol $70 \%$ and subsequently embedded in a methylmethacrylate resin. Undecalcified 5 - $\mu \mathrm{m}$-thick sections were stained by the method of Goldner for quantitative histology to determine static bone parameters. All results are reported as measurements in 2 dimensions using nomenclature established by the American Society for Bone and Mineral Research. $^{39}$ Bone analysis was performed in the Laboratory of Pathophysiology of the University of Antwerp, Belgium, using a semiautomatic image analysis program (AxioVision version 4.51, Zeiss, Jena, Germany) running a custom program. Key parameters that were assessed included bone, perimeter of active osteoblasts on osteoid perimeter (Ob.Pm/O.Pm, \%), perimeter of active osteoclasts on eroded perimeter (Oc.Pm/E.Pm, \%), eroded perimeter on bone perimeter (E.Pm/B.Pm, \%), bone area on tissue area (B.Ar/T.Ar, \%), osteoid area on bone area (O.Ar/B.Ar, \%) and osteoid width $(\mu \mathrm{m})$. Fibrosis was scored as present or absent. Osteoid seams less than $2 \mu \mathrm{m}$ in width were not included in primary measurements of osteoid width or area.

Because the absence of tetracycline labeling precluded determination of dynamic parameters, we used the bone area to total tissue area (B.Ar/T.Ar), osteoid area to bone area (O.Ar/B.Ar), and the ratio of osteoblast-covered perimeter to total bone perimeter (Ob.Pm/ B.Pm) as surrogate markers for bone volume, mineralization, and turnover, respectively. Diagnostic cut-off values of these surrogate markers were determined after comparison static bone with dynamic bone parameters in bone biopsies of a separate cohort of tetracycline-labeled patients. ${ }^{40}$

\section{Statistics}

Results were expressed as mean $\pm \mathrm{SD}$ or median (interquartile range), as appropriate. Patients were categorized according to primary renal disease (ADPKD vs. non-ADPKD). Differences between groups were evaluated using the unpaired Student $t$-test for parametric data and the Mann-Whitney $U$ test for nonparametric data. Categorical data were compared between groups using $\chi^{2}$ test. Simple and multivariable linear regression analyses were used to identify independent determinants of circulating sclerostin and FGF23 levels and bone turnover markers. Nonparametric distributed analytes were natural logarithm-transformed to achieve normality for the regression analyses. The SAS version 9.4 software program (SAS Institute, Cary, NC) was used for the statistical analysis. Twosided $P<0.05$ was considered statistically significant.

\section{DISCLOSURE}

All the authors declared no competing interests.

\section{ACKNOWLEDGMENTS}

We thank the centers of the Leuven Collaborative Group for Renal Transplantation, including their clinicians, surgeons, and nursing staff and the patients who participated in the study. We also thank A. Herelixka and $\mathrm{H}$. Borghs for technical assistance with the data extraction.

\section{AUTHOR CONTRIBUTIONS}

PE designed the study, collected the data, supervised the biochemical analyses and wrote the first draft of the manuscript. All co-authors contributed to the analysis of the data and writing of the manuscript. In addition, EC performed part of the biochemical assays.

\section{SUPPLEMENTARY MATERIAL}

Figure S1. Patient disposition. ADPKD, autosomal dominant polycystic kidney disease; DXA, dual energy x-ray absorptiometry. Table S1. Pearson correlation matrix of bone turnover markers (all $P<0.0001$ ).

Supplementary material is linked to the online version of the paper at www.kidney-international.org.

\section{REFERENCES}

1. Ong AC, Devuyst $O$, Knebelmann B, Walz G. Autosomal dominant polycystic kidney disease: the changing face of clinical management. Lancet. 2015;385(9981):1993-2002.

2. Torres VE, Harris PC, Pirson Y. Autosomal dominant polycystic kidney disease. Lancet. 2007;369(9569):1287-1301.

3. Peters DJM, Sandkuijl LA. Genetic heterogeneity of polycystic kidney disease in Europe. Contrib Nephrol. 1992;97:128-139.

4. Hughes J, Ward CJ, Peral B, et al. The polycystic kidney disease 1 (PKD1) gene encodes a novel protein with multiple cell recognition domains. Nat Genet. 1995;10(2):151-160.

5. Mochizuki T, Wu G, Hayashi T, et al. PKD2, a gene for polycystic kidney disease that encodes an integral membrane protein. Science. 1996;272(5266): 1339-1342.

6. Nguyen AM, Jacobs CR. Emerging role of primary cilia as mechanosensors in osteocytes. Bone. 2013;54(2):196-204.

7. Yuan X, Serra RA, Yang S. Function and regulation of primary cilia and intraflagellar transport proteins in the skeleton. Ann N Y Acad Sci. 2015:1335:78-99.

8. Yoder BK, Hou X, Guay-Woodford LM. The polycystic kidney disease proteins, polycystin-1, polycystin-2, polaris, and cystin, are co-localized in renal cilia. J Am Soc Nephrol. 2002;13(10):2508-2516.

9. Temiyasathit $S$, Jacobs $C R$. Osteocyte primary cilium and its role in bone mechanotransduction. Ann N Y Acad Sci. 2010;1192:422-428.

10. Xiao Z, Quarles LD. Physiological mechanisms and therapeutic potential of bone mechanosensing. Rev Endocr Metab Disord. 2015;16(2):115-129.

11. Xiao Z, Dallas M, Qiu N, et al. Conditional deletion of Pkd1 in osteocytes disrupts skeletal mechanosensing in mice. FASEB J. 2011;25(7): 2418-2432. 
12. Xiao Z, Zhang S, Mahlios J, et al. Cilia-like structures and polycystin-1 in osteoblasts/osteocytes and associated abnormalities in skeletogenesis and Runx2 expression. J Biol Chem. 2006;281(41):30884-30895.

13. Gitomer BY, Pereira RC, Malaczewski MR, et al. Identification of a bone defect in ADPKD. Oral abstract presented at Kidney Week of the American Nephrology Society, November 11-16, 2014; Philadelphia, PA.

14. Gitomer BY, Stoneback JW, Pereira RC, et al. Bone mineral density is reduced in patients with autosomal dominant polycystic kidney disease. Poster abstract presented at Kidney Week of the American Nephrology Society, November 15-20, 2016; Chicago, IL.

15. Moorhead JF, Tatler GL, Baillod RA, et al. Effects of age, sex, and polycystic disease on progressive bone disease of renal failure. Br Med J. 1974;4(5944):557-560.

16. Torres PU, Bover J, Mazzaferro S, et al. When, how, and why a bone biopsy should be performed in patients with chronic kidney disease. Semin Nephrol. 2014;34(6):612-625.

17. Frost ML, Compston JE, Goldsmith D, et al. (18)F-fluoride positron emission tomography measurements of regional bone formation in hemodialysis patients with suspected adynamic bone disease. Calcif Tissue Int. 2013;93(5):436-447.

18. De Rechter S, Bacchetta J, Godefroid N, et al. Evidence for bone and mineral metabolism alterations in children with autosomal dominant polycystic kidney disease. J Clin Endocrinol Metab. 2017;102(11): 4210-4217.

19. Jankowska M, Haarhaus $M$, Qureshi AR, et al. Sclerostin horizontal line A debutant on the autosomal dominant polycystic kidney disease scene? Kidney Int Rep. 2017;2(3):481-485.

20. Gitomer BY, Wolf MS, Salusky IB, et al. Low serum levels of alkaline phosphatase are associated with greater total kidney volume in patients with early autosomal dominant polycystic kidney disease. Poster abstract presented at Kidney Week of the American Nephrology Society, November 15-20, 2016; Chicago, IL.

21. Baron R, Kneissel M. WNT signaling in bone homeostasis and disease: from human mutations to treatments. Nat Med. 2013;19(2):179-192.

22. Spatz JM, Wein MN, Gooi JH, et al. The Wnt inhibitor sclerostin is upregulated by mechanical unloading in osteocytes in vitro. J Biol Chem. 2015;290(27):16744-16758.

23. Corbit KC, Shyer AE, Dowdle WE, et al. Kif3a constrains beta-catenindependent Wnt signalling through dual ciliary and non-ciliary mechanisms. Nat Cell Biol. 2008;10(1):70-76.

24. Raptis V, Bakogiannis C, Loutradis C, et al. Levels of endocan, angiopoietin-2, and hypoxia-inducible factor-1a in patients with autosomal dominant polycystic kidney disease and different levels of renal function. Am J Nephrol. 2018:47(4):231-238.

25. Chen D, Li Y, Zhou Z, et al. HIF-1alpha inhibits Wnt signaling pathway by activating Sost expression in osteoblasts. PLoS One. 2013;8(6):e65940.
26. Cejka D, Herberth J, Branscum AJ, et al. Sclerostin and Dickkopf-1 in renal osteodystrophy. Clin J Am Soc Nephrol. 2011;6(4):877-882.

27. Drechsler C, Evenepoel P, Vervloet MG, et al. High levels of circulating sclerostin are associated with better cardiovascular survival in incident dialysis patients: results from the NECOSAD study. Nephrol Dial Transplant. 2014;30(2):288-293.

28. Viaene L, Behets GJ, Claes K, et al. Sclerostin: another bone-related protein related to all-cause mortality in haemodialysis? Nephrol Dial Transplant. 2013;28(12):3024-3030.

29. Ryan ZC, Ketha H, McNulty MS, et al. Sclerostin alters serum vitamin D metabolite and fibroblast growth factor 23 concentrations and the urinary excretion of calcium. Proc Natl Acad Sci U S A. 2013;110(15): 6199-6204.

30. Bala Y, Farlay D, Boivin G. Bone mineralization: from tissue to crystal in normal and pathological contexts. Osteoporos Int. 2013;24(8): 2153-2166.

31. Naylor KL, Jamal SA, Zou G, et al. Fracture incidence in adult kidney transplant recipients. Transplantation. 2016;100(1):167-175.

32. Gitomer BY, Dalrymple LS, You Z, et al. Fracture rates and post-discharge outcomes among patients undergoing hemodialysis across etiologies of kidney diseases. Poster abstract presented at Kidney Week of the American Nephrology Society, October 31-November 5, 2017; New Orleans, LA.

33. Pavik I, Jaeger P, Ebner L, et al. Soluble Klotho and autosomal dominant polycystic kidney disease. Clin J Am Soc Nephrol. 2012;7(2):248-257.

34. Bouillon R, Van Herck E, Jans I, et al. Two direct (nonchromatographic) assays for 25-hydroxyvitamin D. Clin Chem. 1984;30(11):1731-1736.

35. Bouillon R, De Moor P, Baggiolini EG, Uskokovic MR. A radioimmunoassay for 1,25-dihydroxycholecalciferol. Clin Chem. 1980;26(5):562-567.

36. Bouillon R, Coopmans W, Degroote DE, et al. Immunoradiometric assay of parathyrin with polyclonal and monoclonal region-specific antibodies. Clin Chem. 1990;36(2):271-276.

37. Cavalier E, Souberbielle JC, Gadisseur R, et al. Inter-method variability in bone alkaline phosphatase measurement: clinical impact on the management of dialysis patients. Clin Biochem. 2014;47(13-14): 1227-1230.

38. Behets GJ, Spasovski G, Sterling LR, et al. Bone histomorphometry before and after long-term treatment with cinacalcet in dialysis patients with secondary hyperparathyroidism. Kidney Int. 2015;87(4): 846-856.

39. Dempster DW, Compston JE, Drezner MK, et al. Standardized nomenclature, symbols, and units for bone histomorphometry: a 2012 update of the report of the ASBMR Histomorphometry Nomenclature Committee. J Bone Miner Res. 2013;28(1):2-17.

40. Viaene L, Behets GJ, Heye S, et al. Inflammation and the bone-vascular axis in end-stage renal disease. Osteoporos Int. 2016;27(2):489-497. 